\title{
Cost-Effectiveness of Colorectal Cancer Screening Protocols in Urban Chinese Populations
}

\author{
Weidong Huang ${ }^{1}$, Guoxiang Liu ${ }^{1 *}$, Xin Zhang ${ }^{1}$, Wenqi Fu' ${ }^{1}$, Shu Zheng ${ }^{2 *}$, Qunhong Wu ${ }^{1 *}$, Chaojie Liu ${ }^{3}$, \\ Yang Liu ${ }^{1}$, Shanrong Cai ${ }^{2}$, Yanqin Huang ${ }^{2}$
}

1 School of Health Management, Harbin Medical University, Nangang District, Harbin, China, 2 Cancer Institute (Key Laboratory of Cancer Prevention and Intervention, Ministry of Education of China; Key Laboratory of Molecular Biology in Medical Sciences, Zhejiang Province, China), The Second Affiliated Hospital, Zhejiang University School of Medicine, Hangzhou, China, 3 School of Public Health, La Trobe University, Melbourne, Victoria, Australia

\begin{abstract}
Colorectal cancer (CRC) takes a second and fourth position in the incidence and mortality lists respectively among all malignant tumors in urban populations in China. This study was designed to evaluate the cost-effectiveness of two different CRC screening protocols: faecal occult blood test (FOBT) alone, and FOBT plus a high-risk factor questionnaire (HRFQ) as the respective initial screens, followed by colonoscopy. We developed a Markov model to simulate the progression of a cohort of 100,000 average risk asymptomatic individuals moving through a defined series of states between the ages of 40 to 74 years. The parameters used for the modeling came from the CESP (Comparison and Evaluation of Screening Programs for Colorectal Cancer in Urban Communities in China) study and published literature. Eight CRC screening scenarios were tested in the Markov model. The cost-effectiveness of CRC screening under each scenario was measured by an incremental cost-effectiveness ratio (ICER) compared with a scenario without CRC screening. The study revealed that a combined use of FOBT and HRFQ is preferable in CRC screening programs as an initial screening instrument. Annual FOBT+HRFQ screening is recommended for those who have a negative initial result and those who have a positive result but have failed to continue to colonoscopic examination. Repeated colonoscopy (for those with a positive result in initial screening but a negative colonoscopy result) should be performed at a ten-year interval instead of one-year. Such a protocol would cost 7732 Yuan per life year saved, which is the most cost-effective option. In conclusion, the current Chinese Trial Version for CRC Screening Strategy should be revised in line with the most cost-effective protocol identified in this study.
\end{abstract}

Citation: Huang W, Liu G, Zhang X, Fu W, Zheng S, et al. (2014) Cost-Effectiveness of Colorectal Cancer Screening Protocols in Urban Chinese Populations. PLoS ONE 9(10): e109150. doi:10.1371/journal.pone.0109150

Editor: Helge Bruns, University Hospital Heidelberg, Germany

Received May 4, 2014; Accepted August 29, 2014; Published October 6, 2014

Copyright: (c) 2014 Huang et al. This is an open-access article distributed under the terms of the Creative Commons Attribution License, which permits unrestricted use, distribution, and reproduction in any medium, provided the original author and source are credited.

Data Availability: The authors confirm that all data underlying the findings are fully available without restriction. All relevant data are within the paper and its Supporting Information files.

Funding: This investigation was supported by a special fund to Local Public Health by the China National Fiscal Supplement National Cancer Early Detection and Treatment Program (2006-2009, Ministry of Health and Ministry of Finance, China). The study was partially funded by Health and Family Planning Commission of Heilongjiang Province (project NO. 2012-776) and Education Office of Heilongjiang province (project NO. 12531373). The funders had no role in study design, data collection and analysis, decision to publish, or preparation of the manuscript.

Competing Interests: The authors have declared that no competing interests exist.

* Email: Igx6301@163.com (GL); zhengshu@zju.edu.cn (SZ); wuqunhong@163.com (QW)

\section{Introduction}

Colorectal cancer (CRC) is one of the most prevalent cancers in the world [1]. With high levels of incidence and mortality, GRC imposes a significant and potentially avoidable public health burden in most industrialized countries [2], including the United States, Australia and European countries [3-5]. In China, CRC has attracted increasing attention over recent years, taking a second and fourth position in the incidence and mortality lists respectively among all malignant tumors in urban populations [6]. The National Plan for Cancer Prevention and Control in China (2004-2010) identified CRC as one of the highest priorities for intervention [7].

CRC is characterized by high prevalence, a long asymptomatic period and eminently treatable precancerous lesions, which together suggests that screening is a prudent option. It has been reported in the literature that CRC screening can reduce mortality effectively and even curb incidence as a consequence of polyp removal [8].
There are several protocols already in existence regarding population CRC screening: the most common interventions being Faecal Occult Blood Test (FOBT), flexible sigmoidoscopy, and colonoscopy. The effectiveness of FOBT has been established by randomized clinical trials [9], and population-based screening using FOBT can reduce mortality by one third [10,11]. The European Community and United State Multi-Society Task Force on CRC recommend an annual FOBT as one of multiple options for screening individuals at average risk of CRC [12,13]. The Asia Pacific Working Group Consensus Guideline (APWGCG) recommends FOBT as the first choice for CRG screening in resourcelimited countries [14]. However, using FOBT alone as a screening instrument may fail to detect lesions due to intermittent bleeding from CRC and precancerous polyps or in circumstances where small colorectal neoplasia have little or no tendency for bleeding.

Based on a series of CRC screening efficacy studies [15-17], the Ministry of Health of China proposed a two-step protocol for population-based CRC screening: (1) an initial FOBT and highrisk factor questionnaire (HRFQ) followed by (2) a full colonos- 
copy for those suspected cases identified from the initial screening [18]. Arguably, the choice of CRC screening protocols in resource limited settings should be predicated upon evidence of costeffectiveness considering a wide range of factors such as sensitivity, specificity, acceptability, feasibility, affordability, compliance, and clinical capacity. Many countries such as the USA, Australia, Europe and some Asian countries have sought economic evaluation of their chosen screening protocols for CRC [19]. To our knowledge no such study in mainland China has yet been reported to date (despite extensive enquiry).

In this study, we evaluated the cost-effectiveness of two different CRC initial screening strategies (FOBT vs FOBT+HRFQ) using the Markov model.

\section{Materials and Methods}

\section{Ethics Statement}

This study was approved by the Institutional Review Board of Clinical Research, the Second Affiliated Hospital, Zhejiang University School of Medicine, and was completed in accordance with the ethical principles of the declaration of Helsinki. Written informed consent was sought and obtained from participants prior to the study.

\section{Study Design}

Data for this study came from the project "Comparison and Evaluation of Screening Programs for Colorectal Cancer in Urban Communities in China" (CESP) and published literature. The CESP project was undertaken from July 2006 to December 2008. A total of 400,000 urban residents aged from 40 to 74 years in Hangzhou, Shanghai and Harbin were approached by their local CDC (Center for Disease Control and Prevention) officials, who explained the study to them in detail. Those who agreed to participate in the study were asked to take a FOBT test and fill in a HRFQ. Individuals having one or more of the following features were identified as "risk positive" by the HRFQ: (1) first-degree relative(s) with CRC; (2) a personal history of cancers or intestinal polyps; (3) two or more of the symptoms/histories: (3a) chronic diarrhea; (3b) chronic constipation; (3c) mucous and bloody stool; (3d) history of appendicitis or appendectomy; (3e) history of chronic cholecystitis or cholecystectomy; (3f) history of psychological trauma (e.g. divorce, death of relatives). The participants with either a positive FOBT or a positive HRFQ were offered colonoscopic examination. Any polyps detected during the colonoscopy were removed immediately and sent for histological diagnosis by a pathologist. Those participants who had polyps removed were initially counselled and then followed up three years later with another colonoscopy. The "positive" participants without detected polyps had a second FOBT and HRFQ one year after the initial colonoscopy. Participants with a negative FOBT and those who did not undertake a FOBT screening or colonoscopy were monitored through a routine cancer registry system. Cancers diagnosed by medical facilities are reported to the cancer registry system.

\section{CRC screening protocols tested in this study}

We compared two initial screening protocols: (1) FOBT alone and (2) FOBT plus HRFQ. In both protocols, individuals who were considered of interest were offered a colonoscopic examination.

\section{(1) FOBT as an initial screening instrument}

Four scenarios were developed for protocol one (Figure S1).
Scenario $\mathbf{A}_{1}$ : The participants take a FOBT. Those with a FOBT positive result are offered a colonoscopy. Polyps (if found) are removed during the colonoscopic examination and follow-up colonoscopy is undertaken every three years for those with polyps removed. Those participants without polyps are offered another FOBT in ten years. Participants with an initial negative FOBT result or those having an initial positive FOBT but for whatever reasons elect not to comply with the recommended procedures were offered an annual follow-up FOBT.

Scenario $\mathbf{A}_{2}$ : Similar to Scenario $A_{1}$; however, those participants with an initial negative FOBT result or those having a positive FOBT initially but for whatever reasons elect not to comply with the recommended procedures were monitored through a routine cancer registry system.

Scenario $\mathbf{A}_{3}$ : Similar to Scenario $A_{1}$; however, those participants without polyps take part in an annual follow-up colonoscopy instead of a 10 year interval.

Scenario $\mathbf{A}_{4}$ : Similar to Scenario $A_{3}$; the only difference is that the participants with an initial negative FOBT result or those having a positive FOBT initially but for whatever reasons elect not to comply with the recommended procedures were monitored through a routine cancer registry system.

\section{(2) FOBT plus HRFQ as an initial screening instrument}

Four scenarios were developed for protocol two (Figure S2).

Scenario $\mathbf{B}_{1}$ Participants are offered a FOBT and a HRFQ. Those resulting in a positive outcome (either FOBT or HRFQ) are offered a colonoscopic examination. The follow-up procedures are similar to those of Scenario $A_{1}$.

Scenario $\mathbf{B}_{2}$ Similar to Scenario $B_{1}$; however, participants with an initial negative result(both FOBT and HRFQ)or those with a positive initial result but for whatever reasons elect not to comply with the recommended procedures were monitored through a routine cancer registry system.

Scenario $\mathbf{B}_{3}$ Similar to Scenario $B_{1}$; however, participants without polyps are offered an annual follow-up colonoscopy instead of a 10 years interval.

Scenario $\mathbf{B}_{4}$ This is the scenario currently implemented in China. Participants are offered a FOBT and a HRFQ. Those with a positive result (either FOBT or HRFQ) are offered a colonoscopic examination. Polyps (if present) are removed during the colonoscopic examination and follow-up colonoscopy is undertaken every three years. Those without polyps take part in annual follow-up FOBT and HRFQ. The participants with a negative result(both FOBT and HRFQ)initially and those having a positive result initially but for whatever reasons elect not to comply with the recommended procedures were monitored through a routine cancer registry system.

\section{Markov Model}

We estimated costs and effectiveness of these eight scenarios using the Markov model, a transitional probability model. The Markov model allows us to simulate the trajectory of a hypothetical cohort through different health states [20]. A Markov model describes the probabilities of particular transitions of a particular group of people from one health state to another over a defined period of time. The health states are divided into transient states and absorbing states. A transient state can change to another transient state or to an absorbing state; whereas an absorbing state (such as death) cannot change to other states (such as normal, polyp, CRC) [20]. We developed the Markov model using Microsoft Excel to simulate the progression of a cohort of 100,000 average-risk asymptomatic individuals moving through a 
Table 1. Parameters used for the modeling of CRC screening protocols.

\begin{tabular}{|c|c|c|}
\hline Variable & Values (range) & Ref. \\
\hline Sensitivity of FOBT & $42.90 \%(20 \%-60 \%)$ & {$[16,22]$} \\
\hline Sensitivity of FOBT+HRFQ & $88.90 \%(75 \%-90 \%)$ & {$[16,22]$} \\
\hline Specificity of FOBT & $86.10 \%(50 \%-90 \%)$ & {$[16,22]$} \\
\hline Specificity of FOBT+HRFQ & $71.70 \%(50 \%-90 \%)$ & {$[16,22]$} \\
\hline Coverage of FOBT & $45.37 \%(30 \%-100 \%)$ & CESP \\
\hline Compliance with colonoscopy request after initial screening by FOBT & $37.32 \%(30 \%-100 \%)$ & CESP \\
\hline Coverage of FOBT plus HRFQ & $53.22 \%(30 \%-100 \%)$ & CESP \\
\hline Compliance with colonoscopy request after initial screening by FOBT+HRFQ & $46.78 \%(30 \%-100 \%)$ & CESP \\
\hline Polypectomy in people screened by FOBT & $32.07 \%$ & CESP \\
\hline Polypectomy in people screened by FOBT+HRFQ & $26.13 \%$ & CESP \\
\hline CRC prevented by colonoscopy & $75 \%$ & [21] \\
\hline Discount rate & $3 \%(0 \%-7 \%)$ & [24] \\
\hline \multicolumn{3}{|l|}{ Cost (Yuan) } \\
\hline Marketing for FOBT & 1 & CESP \\
\hline Marketing for FOBT+HRFQ & 1 & CESP \\
\hline Material of FOBT & 5 & CESP \\
\hline Material of FOBT+HRFQ & 7 & CESP \\
\hline Distribution and return of FOBT & 3 & CESP \\
\hline Distribution and return of FOBT+HRFQ & 3 & CESP \\
\hline Pathology & 150 & $\mathrm{BNHI}$ \\
\hline Colonoscopy & 290 & $\mathrm{BNHI}$ \\
\hline Polypectomy & 500 & $\mathrm{BNHI}$ \\
\hline Treatment of CRC & 41602 & BNHI \\
\hline
\end{tabular}

defined series of states from 40 to 74 years. In this simulation, the health states of individuals were categorized either as normal, polyp, CRG or death. After successive iterations, the model estimated the cumulative costs and effectiveness for the entire cohort over a35 year period. Each resultant simulation was compared with that of a scenario in which no screening is involved.

\section{Transitional Parameters}

The simulation model was developed using Chinese population data. Some transitional parameters were borrowed from studies in other countries if they were not available in China.

The CESP project provided most of the clinical, epidemiological and costing data. It revealed that $45.37 \%$ and $53.22 \%$ eligible participants complied with the initial FOBT and FOBT+HRFQ requests respectively. Some $37.32 \%$ FOBT positive participants and $46.78 \%$ FOBT+HRFQ positive participants accepted the offer of colonoscopy. Every participant with polyps had polypectomy, amongst whom $32.07 \%$ resulted from FOBT screening alone, and $26.13 \%$ from FOBT+HRFQ screening. Previous studies showed that colonoscopic polypectomy can probably reduce CRG incidence by around 76-90\% [21]. For this study, we assumed a conservative reduction of $75 \%$ CRC incidence following colonoscopic polypectomy. The sensitivity and specificity of FOBT were found to be $42.90 \%$ and $86.10 \%$, respectively. The sensitivity of FOBT+HRFQ increased to $88.90 \%$, while its specificity decreased to $71.70 \% \quad[16,22]$. The incidence and fatality data used in the simulation model came from the Chinese Cancer Registry Annual Reports [6] and the 5th National Census [23] (Table 1).

\section{Cost estimates}

Only direct costs were estimated in this study by the third-party payer's perspective, which included costs associated with initial screening, colonoscopy, polypectomy, pathology tests, and treatment of CRC. The initial screening costs comprised expenses in marketing, materials and reagents for FOBT and HRFQ and distribution and return of FOBT and HRFQ. These were calculated using the GESP data. All other costs were calculated based on the claim data of the Bureau of National Health Insurance (BNHI). All costs are expressed in Chinese Yuan in this paper and are inflated to the 2008 price level.

\section{Effectiveness of CRC screening}

The effectiveness of CRC screening was presented in terms of "Life Years" saved by the screening. It was calculated through estimating premature deaths (from 40 to 74 years old) as a result of CRC using an age-dependent formula for each age group. The "Life Years" saved under each screening scenario equals to the difference in life years lost between the tested screening scenario and the scenario without any screening. In this study, discount rates for both future costs and future life years were set at 3\% [24]. 


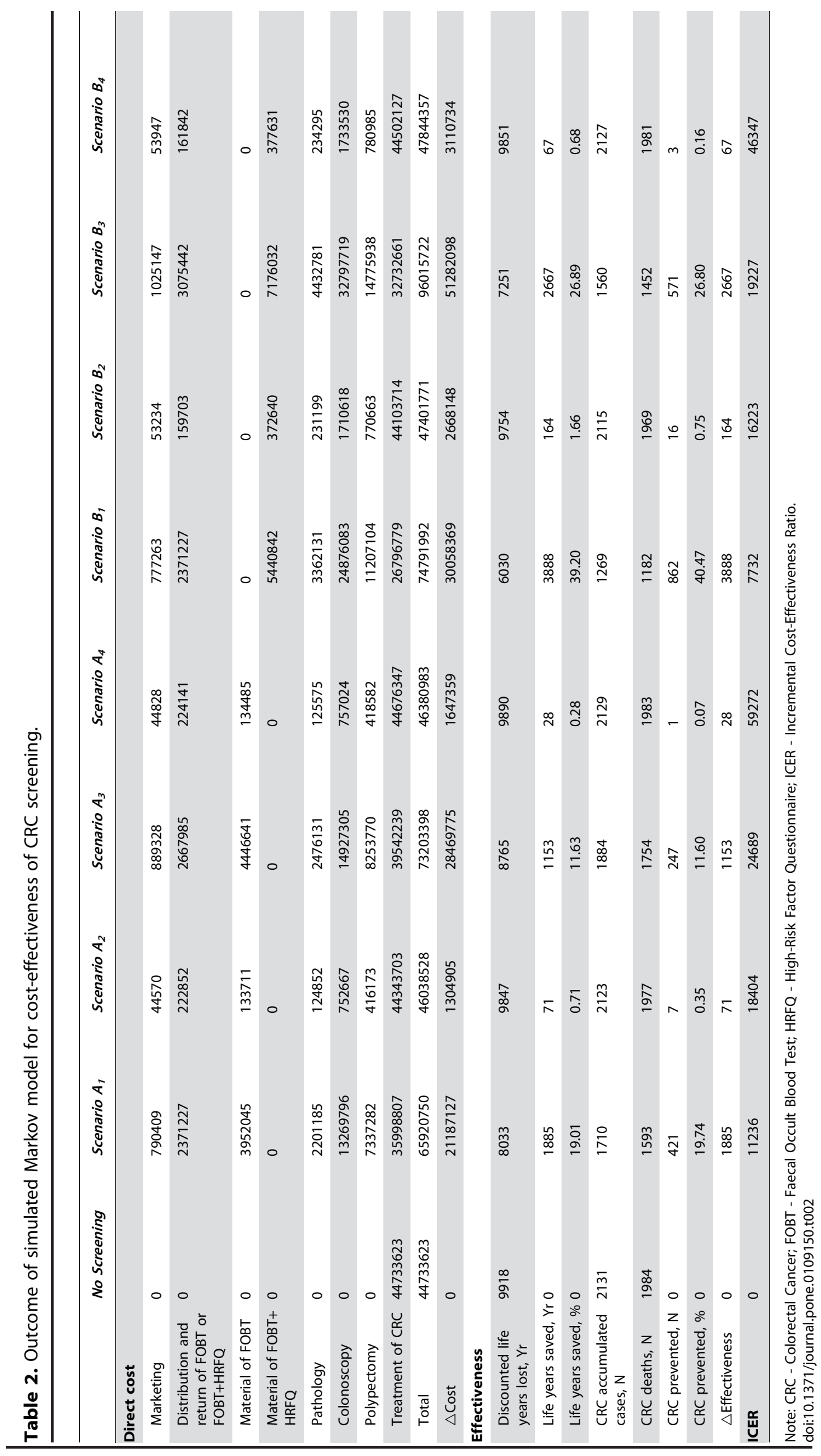




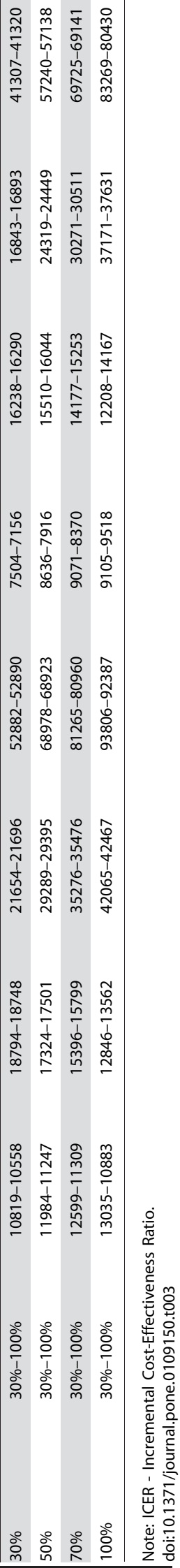

\section{Cost-effectiveness indicator}

We used an Incremental cost-effectiveness ratio (ICER) to measure the cost-effectiveness of the tested screening protocols, defined as the "difference in costs divided by the corresponding difference in effectiveness". A smaller ICER indicates lower cost for saving one life year, reflecting improved cost-effectiveness.

\section{Sensitivity analysis}

In the sensitivity analysis, we tested the impact of several parameters such as compliance, sensitivity, specificity, and discount rate on the robustness of the simulation model. Oneway and two-way sensitivity analyses were applied to assess the influence of those parameters on ICER. The ranges of parameter variations were set as: FOBT - $30 \%$ to $100 \%$ for compliance; $20 \%$ to $60 \%$ for sensitivity; and $50 \%$ to $90 \%$ for specificity; FOBT plus HRFQ - $30 \%$ to $100 \%$ for compliance; $75 \%$ to $90 \%$ for sensitivity; and $50 \%$ to $90 \%$ for specificity; Colonoscopy $-30 \%$ to $100 \%$ for compliance; Discount rate - $0 \%$ to $7 \%$ (Table 1 ).

\section{Results}

\section{Costs}

When no screening was performed, the accumulated expenses over 35 years were estimated through 35 successive iterations in Markov modeling, which resulted in a total of 44,733,623 Yuan for 100,000 average-risk asymptomatic individuals aged 40 years. The total costs under the screening scenario would increase compared to that without screening, with Scenario $A_{2}$ having the lowest and Scenario $B_{3}$ having the highest costs. Nevertheless, CRG treatment costs were lower under all screening scenarios compared with those without screening (Table 2).

\section{Effectiveness}

The simulation identified 2131 cases of CRC when no screening was adopted, representing a loss of 9918 CRC-related discounted life years: screening prevents CRC and reduces the loss of CRCrelated life years. The highest level of effectiveness was achieved under Scenario $B_{1}$, which reduced $40.47 \%$ (862 cases) of CRC and avoided $39.20 \%$ of loss of CRC-related life years (3888 discounted life years) compared with those without screening (Table 2).

\section{Costs-effectiveness}

For every life year saved,7732 Yuan would be needed under Scenario $B_{1}, 11,236$ Yuan under Scenario $A_{1}, 18,404$ Yuan under Scenario $A_{2}, 24,689$ Yuan under Scenario $A_{3}, 59,272$ Yuan under Scenario $A_{4}, 16,223$ Yuan under Scenario $B_{2}, 19,227$ Yuan under Scenario $B_{3}$, and 46,347 Yuan under Scenario $B_{4}$.Scenario $B_{1}$ is the most cost-effective protocol among all the scenarios.

\section{Sensitivity analysis}

A greater change in ICER was found when colonoscopy request compliance increased compared with that when coverage of initial screening increased. Colonoscopy compliance also mediated the impact of initial screening coverage on ICER. ICER was more sensitive to changes in initial screening coverage when colonoscopy compliance was higher (Table 3 ).

ICER decreased with rising sensitivity of initial screening. Scenario $A_{3}$ and $A_{4}$ were more sensitive to changes in FOBT sensitivity than Scenario $A_{1}$ and $A_{2}$. When FOBT sensitivity surpassed $42.9 \%$ (the parameter used in the modeling), changes in ICER had slowed down dramatically. Moderate changes in ICER 


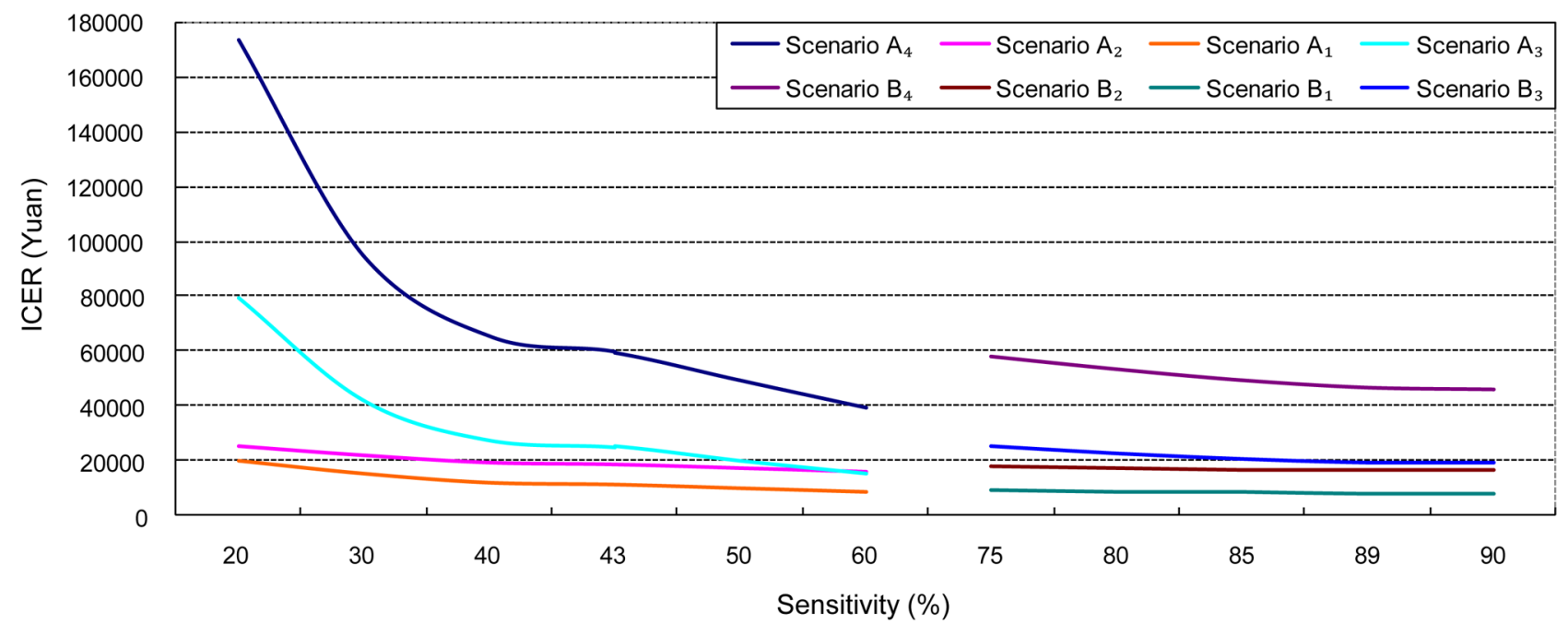

Figure 1. ICER decreases with rising sensitivity of FOBT or FOBT+HRFQ: One-way sensitivity analysis. doi:10.1371/journal.pone.0109150.g001

were found when the sensitivity of FOBT+HRFQ increased (Figure 1).

Similarly, ICER decreased with rising specificity of initial screening. Scenario $A_{3}$ and $A_{4}$ were more sensitive to changes in specificity of initial screening than other scenarios. When the specificity of initial screening surpassed $86.1 \%$ for FOBT or $71.7 \%$ for FOBT+HRFQ (the parameters used in the modeling), changes in ICER had slowed down dramatically (Figure 2).

ICER increased with rising discount rate. The ranking order of the eight scenarios in ICER remained largely unchanged with the increase of discount rate, except for Scenario $B_{3}$. Scenario $B_{3}$ was less sensitive to rising discount rate than the others (Figure 3).

Scenario $B_{1}$ proved to be the most cost-effective, regardless of how the above mentioned parameters changed.

\section{Discussion}

The Markov model simulation revealed that Scenario $B_{1}$ is the most cost-effective protocol for CRC screening, followed by Scenario $A_{1}, B_{2}, A_{2}, B_{3}, A_{3}, B_{4}$ and $A_{4}$. The cost per life year saved under Scenario $B_{1}$ is the lowest, regardless how simulation parameters were set or changed.

This finding indicates that a combined use of FOBT and HRFQ as an initial step for CRC screening is a better strategy than FOBT alone. Although this means an increase of costs, a greater level of effectiveness can be achieved. This study demonstrated that the costs of protocol two (FOBT+HRFQ as initial screening) under different scenarios are consistently higher than those of protocol one (FOBT as initial screening) under corresponding scenarios (i.e. $A_{1} v s B_{1}, A_{2} v s B_{2}, A_{3} v s B_{3}, A_{4} v s B_{4}$ ).

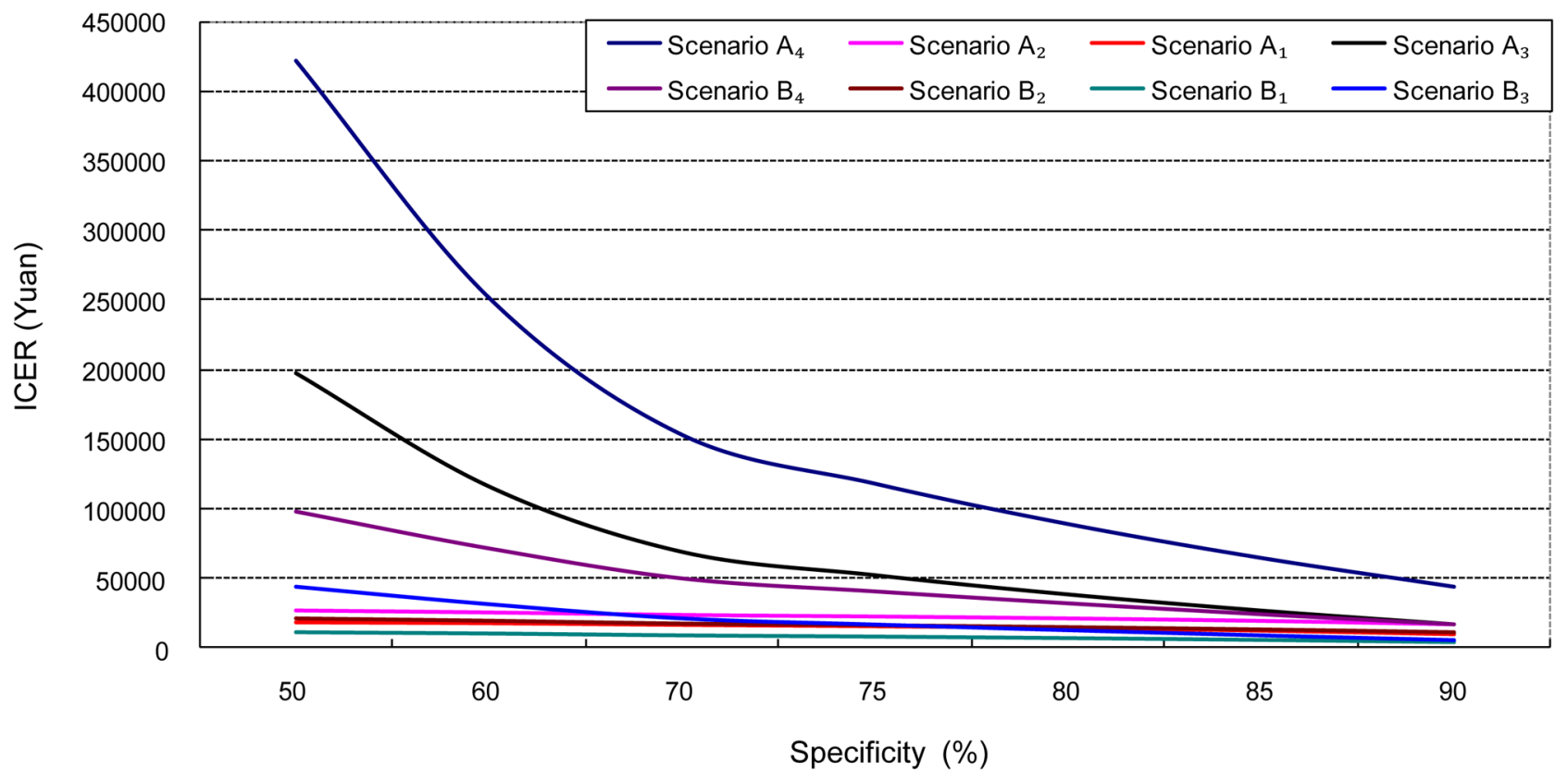

Figure 2. ICER decreases with rising specificity of FOBT or FOBT+HRFQ: One-way sensitivity analysis. doi:10.1371/journal.pone.0109150.g002 


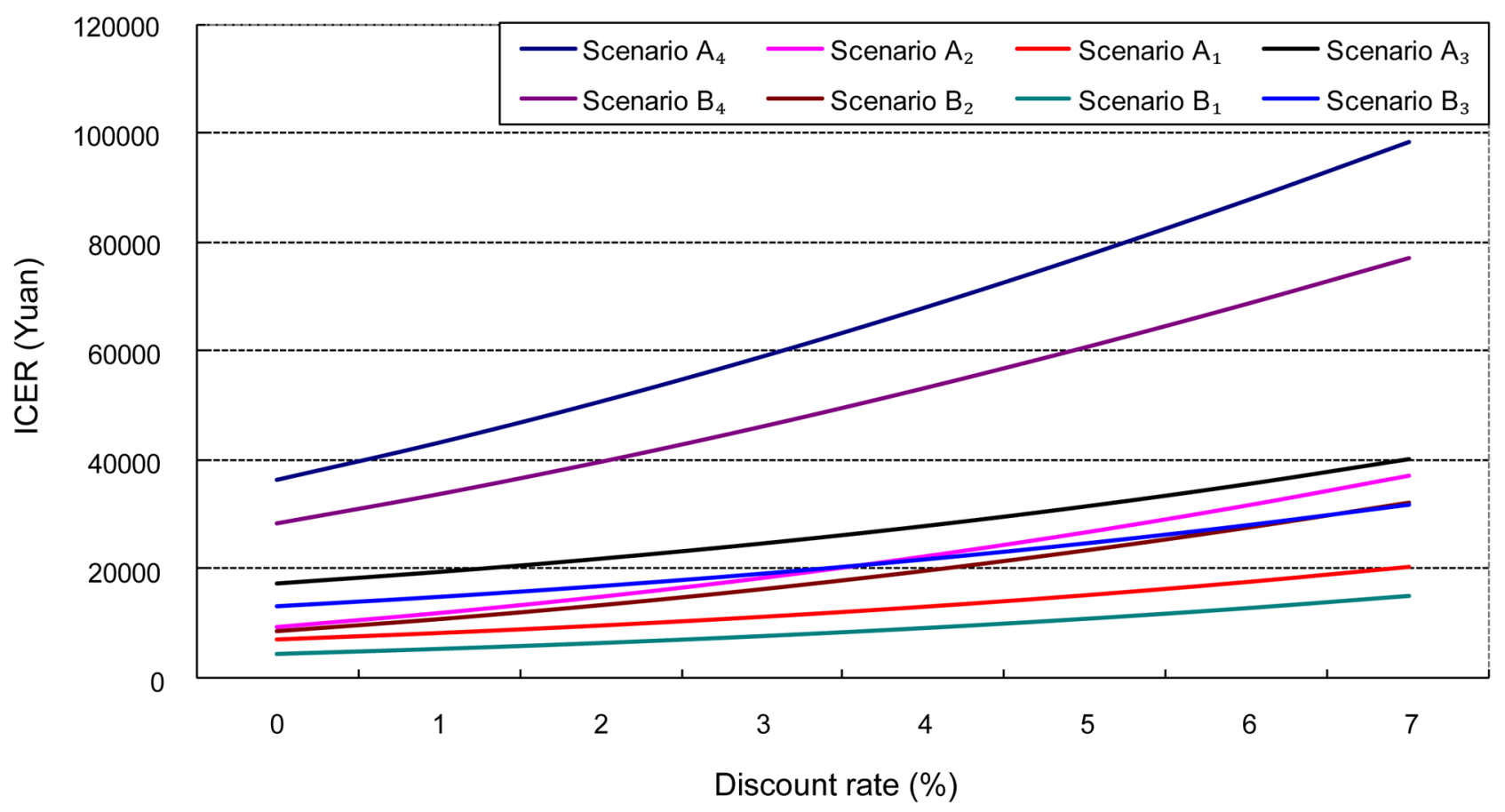

Figure 3. ICER increases with discount rate: One-way sensitivity analysis. doi:10.1371/journal.pone.0109150.g003

However, the effectiveness of protocol two is consistently better than that of protocol one. In addition, regardless how simulation parameters were set or changed, the ICER of protocol two are always lower than those of protocol one.

For people having an initial negative screening result and those having a positive result but failing to comply with the recommended procedures, repeating the initial screening annually can produce a more cost-effective result than routine cancer registry only. This study showed that, for both protocol one and protocol two, scenarios with a repeated initial screening incurred greater costs consistently compared with their alternative counterparts requesting routine cancer registry only $\left(A_{1}\right.$ vs $A_{2} ; A_{3}$ vs $A_{4} ; B_{1}$ vs $B_{2} ; B_{3}$ vs $\left.B_{4}\right)$. However, the effectiveness and costeffectiveness as measured by ICER of those scenarios with a repeated initial screening are consistently better than their alternative counterparts using routine cancer registry only.

For people having a negative colonoscopy result, a repeated colonoscopy every ten years can produce a more cost-effective result: annual colonoscopy is too expensive. The effectiveness and cost-effectiveness as measured by ICER for repeated colonoscopy at a ten-year interval are consistently better than those with annually repeated colonoscopy under corresponding scenarios $\left(A_{1}\right.$ vs $A_{3} ; A_{2}$ vs $A_{4} ; B_{1}$ vs $B_{3} ; B_{2}$ vs $B_{4}$ ).

Compliance rates have a significantly impact on the total cost and effectiveness of CRC screening programs. In previous studies, compliance rates for FOBT and colonoscopy were often estimated for modeling [25-28]. In this study, we built our models using real observational data. Meanwhile, we tested the impact of compliance rates on the models by varying the rates from $30 \%$ to $100 \%$. We found that the compliance rates of our study participants are lower compared with findings undertaken elsewhere in China [29]. Zheng et al [29] achieved $87.4 \%$ coverage of FOBT+HRFQ screening and $76.6 \%$ compliance for colonoscopy requests in a rural Chinese population, significantly higher than those of this study population. However, under the preferred Scenario $B_{1}$, the cost-effectiveness of the screening program would remain virtually unchanged if similar compliance rates were achieved in our study population because the increase of FOBT+HRFQ coverage would lead to a slight decrease of ICER; whereas, a slight increase of ICER would appear when compliance with colonoscopy increases.

It is unclear what contributed to the low compliance rates for CRC screening in our study population: further studies are warranted. Experiences of developed countries demonstrated that to reduce financial barriers and ensure equal access to those cancer screening programs are better financed by governments [30-33]. Empirical evidence shows that improved understanding of CRC screening can encourage people to comply with prescribed procedures in screening programs [34,35]. Unfortunately, GRC screening guidelines freely available to the public in some developed countries remain unavailable in China.

In China, cervical and breast cancer screening programs have been included in public health services for rural populations since 2009and a good cost-effectiveness has been presented[36,37].Based on evidence support of this study, we suggest that CRC screening be included in the public health services list.

Compared to previous studies, this study has some unique characteristics. It is worthy to note that the combined use of FOBT and HRFQ as initial screening for CRC is an original development in China. To our knowledge, this is the first study of its kind attempting to evaluate the cost-effectiveness of CRC screening programs in urban Chinese populations. The core data used for the simulation modeling came from real observational data.

\section{Limitations}

In this study, we only calculated direct costs. Indirect costs such as those associated with production loss due to attending screening and treatment services should be considered in future studies. 


\section{Conclusion}

A combined use of FOBT and HRFQ is preferable in CRC screening programs as an initial screening instrument. Annual FOBT+HRFQ screening is recommended for those who have a negative initial result and those who have a positive result but have failed to comply with colonoscopy procedures. Repeated colonoscopy (for those with a positive result in initial screening but a negative colonoscopy result) should be performed at a ten-year interval instead of one-year.

The current Chinese Trial Version for CRC Screening Strategy falls into Scenario $B_{4}$, which is one of the least cost-effective options and should be revised in line with Scenario $B_{1}$.

\section{Supporting Information}

Figure S1 Markov process for CRC screening protocol one (Scenario $A_{1}-A_{4}$ ) using FOBT as initial screening procedure. Transitions to different Markov states (in oval) are described, with normal, polyp and CRC as transient states and death as an absorbing state (patients cannot leave). The parameters used in the model were described in Table 1. Note: CRC - Colorectal Cancer; FOBT - Faecal Occult Blood Test; NC - No Compliance.

(TIF)

\section{References}

1. Jemal A, Bray F, Center MM, Ferlay J, Ward E, et al. (2011) Global cancer statistics. CA Cancer J Clin 61(2): 69-90.

2. Micheli A, Mugno E, Krogh V, Quinn MJ, Coleman M, et al. (2002) Cancer prevalence in European registry areas. Ann Oncol 13(6): 840-65.

3. Jemal A, Siegel R, Xu J, Ward E (2010) Cancer statistics, 2010. CA Cancer J Clin 60(5): 277-300.

4. Ferlay J, Autier P, Boniol M, Heanue M, Colombet M, et al. (2007) Estimates of the cancer incidence and mortality in Europe in 2006. Ann Oncol 18(3): 581-92.

5. Ananda SS, McLaughlin SJ, Chen F, Hayes IP, Hunter AA, et al. (2009) Initial impact of Australia's national bowel cancer screening program. Med J Aust 191(7): 378-81.

6. Zhao P, Chen WQ (2011) Chinese Cancer Registry Annual Report 2010 China: Military Medical Science Press 32-38.

7. the Plan for the Cancer Prevention and Control in China (2004-2010). China Cancer 13(2): 65-68.

8. Pignone M, Rich M, Teutsch SM, Berg AO, Lohr KN (2002) Screening for colorectal cancer in adults at average risk: a summary of the evidence for the U.S. Preventive Services Task Force. Ann Intern Med 137(2): 132-41.

9. Towler B, Irwig L, Glasziou P, Kewenter J, Weller D, et al. (1998) A systematic review of the effects of screening for colorectal cancer using the faecal occult blood test, hemoccult. BMJ 317(7158): 559-65.

10. Kronborg O, Fenger C, Olsen J, Jørgensen OD, Søndergaard O (1996) Randomised study of screening for colorectal cancer with faecal-occult-blood test. Lancet 348(9040): 1467-71.

11. Mandel JS, Bond JH, Church TR, Snover DC, Bradley GM, et al. (1993) Reducing mortality from colorectal cancer by screening for fecal occult blood. N Engl J Med 328(19): 1365-71.

12. Winawer S, Fletcher R, Rex D, Bond J, Burt R, et al. (2003) Colorectal cancer screening and surveillance: clinical guidelines and rationale - update based on new evidence. Gastroenterology 124(2): 544-60.

13. Arbyn M, Van Oyen H, Lynge E, Micksche M, Faivre J, et al. (2003) European Commission's proposal for a council recommendation on cancer screening. BMJ 327(7409): 289-90.

14. Sung JJ, Lau JY, Young GP, Sano Y, Chiu HM, et al. (2008) Asia Pacific consensus recommendations for colorectal cancer screening. Gut 57(8): 116676.

15. Zheng S, Chen K, Liu X, Ma X, Yu H, et al. (2003) Cluster randomization trial of sequence mass screening for colorectal cancer. Dis Colon Rectum 46(1): 51-8.

16. Liu XY, Zheng S (1997) An evaluation for optimization colorectal cancer screening in high-risk groups. Cancer Research on Prevention and Treatment 24(4): 197-200.

17. Chen K (1988) A quantification method of colorectal cancer screening. Journal of Zhejiang Medical University 17(2): 49-52.

18. Dong ZW (2009) Guidelines of cancer screening, early detection and early treatment of China (Trial version). Peiking People's Medical Publishing House 123-53.

19. Lansdorp-Vogelaar I, Knudsen AB, Brenner H (2010) Cost-effectiveness of colorectal cancer screening-an overview. Best Pract Res Clin Gastroenterol 24(4): 439-49.
Figure S2 Markov process for CRC screening protocol two (Scenario $B_{1}-B_{4}$ ) using FOBT+HRFQ as initial screening procedure. Transitions to different Markov states (in oval) are described, with normal, polyp and CRC as transient states and death as an absorbing state (patients cannot leave). The parameters used in the model were described in Table 1. Note: CRC - Colorectal Cancer; FOBT+HRFQ -Faecal Occult Blood Test + High-Risk Factor Questionnaire; NG - No Compliance. (TIF)

\section{Acknowledgments}

The authors would like to thank the members of China National Committee on Early Detection and Treatment for Cancers, the general practitioners from participating communities, and the doctors and nurses from the local hospitals who supported the trial. Mr. Adamm Ferrier provided language editing and proofreading on the manuscript.

\section{Author Contributions}

Conceived and designed the experiments: WH GL SZ QW. Performed the experiments: WH. Analyzed the data: WH XZ WF YH. Contributed reagents/materials/analysis tools: GL SZ. Wrote the paper: WH GL SZ QW. Revised the manuscript: CL YL SC.

20. Sonnenberg FA, Beck JR (1993) Markov models in medical decision making a practical guide. Med Decis Making 13(4): 322-38.

21. Winawer SJ, Zauber AG, Ho MN, O’Brien MJ, Gottlieb LS, et al. (1993) Prevention of colorectal cancer by colonoscopic polypectomy. The National Polyp Study Workgroup. N Engl J Med. 329(27): 1977-81.

22. Zheng S, Yu H (1991) The model and evaluation of colorectal cancer screening. Chinese medical journal 71(7): 381-4.

23. Tabulation on the 2000 Population Census of the People's Republic of China (1 volumes) Beijing, China: China Statistics Press.

24. Drummond MF, Jefferson TO (1996) Guidelines for authors and peer reviewers of economic submissions to the BMJ. The BMJ Economic Evaluation Working Party. BMJ 313(7052): 275-83.

25. Sonnenberg A, Delcò F, Inadomi JM (2000) Cost-effectiveness of colonoscopy in screening for colorectal cancer. Ann Intern Med 133(8): 573-84.

26. Wu GH, Wang YM, Yen AM, Wong JM, Lai HC, et al. (2006) Costeffectiveness analysis of colorectal cancer screening with stool DNA testing in intermediate-incidence countries. BMC cancer 6(1): 136.

27. Berchi C, Bouvier V, Réaud JM, Launoy G. (2004) Cost-effectiveness analysis of two strategies for mass screening for colorectal cancer in France. Health Econ 13(3): 227-38.

28. Tsoi KK, Ng SS, Leung MC, Sung JJ (2008) Cost-effectiveness analysis on screening for colorectal neoplasm and management of colorectal cancer in Asia. Aliment Pharmacol Ther 28(3): 353-63.

29. Zheng S, Zhang SZ, Cai SR, Huang YQ (2009) Protocol and Practice for Colorectal Cancer Screening. China Cancer 18(9): 700-4.

30. Masseria C (2010) Colorectal cancer in Italy: a review of current national and regional practice on screening and treatment. Eur J Health Econ 10 (Suppl 1): S41-9.

31. Saito H (2006) Colorectal cancer screening using immunochemical faecal occult blood testing in Japan. J Med Screen (suppl 1): S6-7.

32. Choi KS, Jun JK, Lee HY, Hahm MI, Oh JH, et al. (2010) Increasing uptake of colorectal cancer screening in Korea: a population-based study. BMC Public health 10(1): 265.

33. Ascunce N, Salas D, Zubizarreta R, Almazán R, Ibáñez J, et al. (2010) Cancer screening in Spain. Ann Oncol (Suppl 3): iii43-51.

34. Cai SR, Zhang SZ, Zhu HH, Zheng S (2009) Barriers to colorectal cancer screening: a case-control study. World J Gastroenterol 15(20): 2531-6.

35. Meng W, Bi XW, Bai XY, Pan HF, Cai SR, et al. (2009) Barrier-focused intervention to increase colonoscopy attendance among nonadherent high-risk populations. World J Gastroenterol 15(31): 3920-5.

36. Shi JF, Canfell K, Lew JB, Zhao FH, Legood R, et al. (2011) Evaluation of primary HPV-DNA testing in relation to visual inspection methods for cervical cancer screening in rural China: an epidemiologic and cost-effectiveness modelling study. BMC cancer 11(1): 239.

37. Canfell K, Shi JF, Lew JB, Walker R, Zhao FH, et al. (2011) Prevention of cervical cancer in rural China: evaluation of HPV vaccination and primary HPV screening strategies. Vaccine 29(13): 2487-94. 